\title{
On the Origin of Retrograde Orbit Satellites around Saturn and Jupiter
}

\author{
Yuehua $\mathbf{M a}^{1,2}$, Jiaqing Zheng ${ }^{2,1}$ and Xiaohai Shen ${ }^{3}$ \\ ${ }^{1}$ Purple Mountain Observatory, Nanjing 210008, China, \\ email: yhma@pmo.ac.cn \\ ${ }^{2}$ Turku University, Tuorla Observatory, 21500 Piikkiö, Finland \\ email: zheng@utu.fi \\ ${ }^{3}$ Jiaozuo Teachers College, Henan, 454000, China
}

\begin{abstract}
Many Retrograde Orbit Satellites around Jupiter and Saturn have been found recently. Most of them are small with irregular shapes. They are farther from the planet than regular satellites. Their orbits have big eccetricities.

We tested their dynamical origin and found:

1. The small bodies can be captured by normal satellites and form retrograde orbits. But these orbits are not stable. Sooner or later, they would escape from planetary region or fall down into the planets.

2. Another way is that they have formed by collisions just after regular moons formed. We studied the mechanism and obtained good results.
\end{abstract}

Keywords. Solar System, planets and satellites: formation.

\section{Introduction}

Jupiter and Saturn have $63(\mathrm{R}=48)$ and $61(\mathrm{R}=29)$ moons, respectively (Figure 1, or http://ssd.jpl.nasa.gov). " $\mathrm{R}$ " is the number of retrograde orbits (dark colour in Figure).

We list retrograde irregular moons of Saturn on table 1 and put a photo of Phoebe biggest irregular moon (Figure 1, right side) here. We also list prograde irregular moons on the table since they have irregular shapes and big $i$ and $e$.

From the table and Figures we can see:

(1) Normal moons are very close to their planets and with regular orbits ( $\operatorname{small} i, e)$. They have regular shapes (ball).

(2) Irregular moons are far from their planets with irregular shapes.

(3) Retrograde irregular moons mostly are far from their planet. They are located about half of the radius of planetary activity sphere and their orbits are near planetary orbital plane (few near $145^{\circ}$, most $>165^{\circ}$ ).

(4) Prograde irregular moons are closer than retrograde irregular moons, about $25 \%$ of radius of planetary activity sphere. Their inclinations are big, mostly about $45^{\circ}$.

Normal moons have formed by accretion. This is why they have regular shapes and regular orbits. Irregular moons cannot form by normal process as normal moons. We suppose that they were from outer solar system.

\section{Test and problems of capture process}

In an early work (Zheng 1994), we proved that short period comets were captured when they came from Oort cloud (or Kuiper belt) by close encounters with planets. The capture process of moons can be similar when small bodies come into planetary activity sphere (see Figure 2). 
Table 1. Irregular moons of Saturn

\begin{tabular}{lccccllllll}
\hline Name & $a(k m)$ & $i\left(^{\circ}\right)$ & $e$ & size(km) & & & & & & \\
\hline Kiviuq & 11111000 & 45.71 & 0.334 & 16 & Jarnsaxa & 18811000 & 163.3 & 0.216 & 6 \\
Ijiraq & 11124000 & 46.44 & 0.316 & 12 & Narvi & 19007000 & 145.8 & 0.431 & 7 \\
Phoebe & 12944300 & 174.8 & 0.164 & 240 & Bergelmir & 1933800 & 158.5 & 0.142 & 6 \\
Paaliaq & 15200000 & 45.13 & 0.364 & 22 & Suttungr & 19459000 & 175.8 & 0.114 & 7 \\
Skathi & 15541000 & 152.6 & 0.270 & 8 & Hati & 19856000 & 165.8 & 0.372 & 6 \\
Albiorix & 16182000 & 33.98 & 0.478 & 32 & Bestla & 20129000 & 145.2 & 0.521 & 7 \\
Bebhionn & 17119000 & 35.01 & 0.469 & 6 & Farbauti & 20390000 & 156.4 & 0.206 & 5 \\
Erriapo & 17343000 & 34.62 & 0.474 & 10 & Thrymr & 20474000 & 176.0 & 0.470 & 7 \\
Siarnaq & 17531000 & 45.56 & 0.295 & 40 & Aegir & 20735000 & 166.7 & 0.252 & 6 \\
Skoll & 17665000 & 161.2 & 0.464 & 6 & Kari & 22118000 & 156.3 & 0.478 & 7 \\
Tarvos & 17983000 & 33.82 & 0.531 & 15 & Fenrir & 22453000 & 164.9 & 0.136 & 4 \\
Tarqeq & 18009000 & 46.09 & 0.160 & 7 & Surtur & 22707000 & 177.5 & 0.451 & 6 \\
Greip & 18206000 & 179.8 & 0.326 & 6 & Ymir & 23040000 & 173.1 & 0.335 & 18 \\
Hyrrokkin & 18437000 & 151.4 & 0.333 & 8 & Loge & 23065000 & 167.9 & 0.187 & 6 \\
Mundilfari & 18685000 & 167.3 & 0.210 & 7 & Fornjot & 25108000 & 170.4 & 0.206 & 6 \\
Unnamed & Irregular & moons & & & & & & & \\
S/2004 S07 & 19800000 & 165.1 & 0.580 & 6 & S/2006 S1 & 18981135 & 154.2 & 0.130 & 6 \\
S/2004 S12 & 19650000 & 164.0 & 0.401 & 5 & S/2006 S3 & 21132000 & 150.8 & 0.471 & 6 \\
e S/2004 S13 & 18450000 & 167.4 & 0.273 & 6 & S/2007 S2 & 16560000 & 176.7 & 0.218 & 6 \\
e S/2004 S17 & 18600000 & 166.6 & 0.259 & 4 & S/2007 S3 & 20518500 & 177.2 & 0.130 & 5 \\
\hline
\end{tabular}

Notes: We put some prograde orbit moons on the table since they are also "Irregular" by shapes and big e.

Retrograde orbit moons can be captured by normal moons of the planets, but these orbits always cross the orbits of normal moons, then sooner or later, they would have new close encounters which could cause them to escape. These orbits are not stable. In other words, if retrograde orbit moons were captured by inner moons of Saturn, they can be the transfer source of short period comets, but they may not become into stable orbits as current retrograde moons we see now.
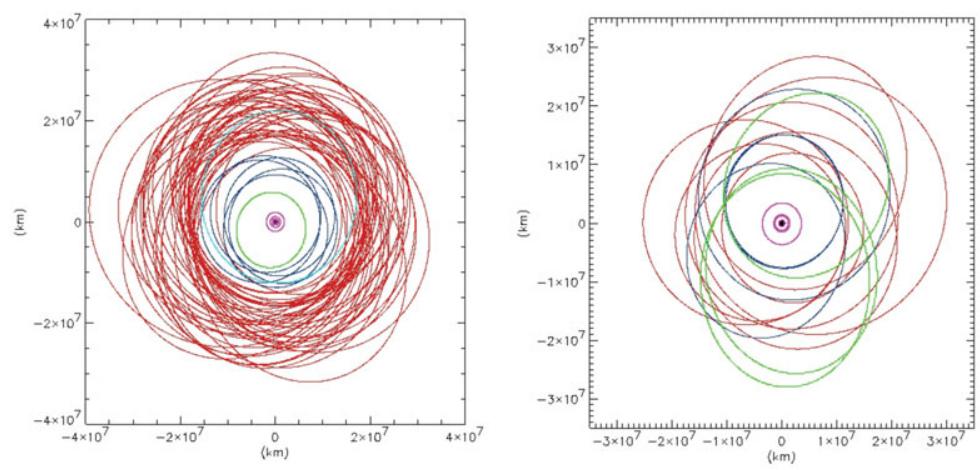

Figure 1. Jupiter and Saturn's Known Satellites

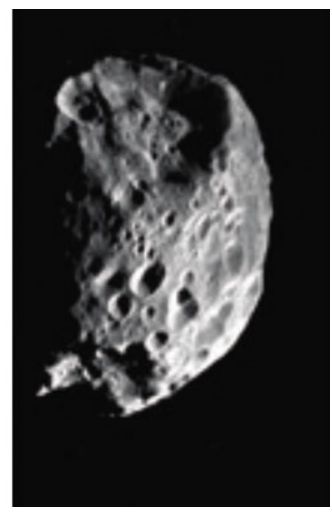

Phoebe (by Cassini) 


\section{Collision process - formation of retrograde orbits}

Now, we study retrograde moons of Saturn (similar for Jupiter). Since current orbits of retrograde moons are stable, they might form by another way at early time after Saturn formed. At that time, around Saturn's orbit, still there were some planetesimals, and around Saturn, after normal moons formed, many small bodies remained also. When these two kinds bodies met in Saturn's activity area, the relative motion between them were opposite: planetesimals were "retrograde" and small bodies around Saturn were prograde, when they collided or combined with each other, some retrograde moons could form.

Let's consider a small body moves with a planet, the planet moves on a circular orbit and small body has eccentricity $0.1: a_{p}=1, e_{p}=0, a_{s}=1, e_{s}=0.1$.

In Figure 3, left side is the orbits of planet and the small body in a fixed coordinate, right side is the orbits on a rotating coordinate. In a rotating coordinate, the small body moves around the planet looks like a satellite but moves on a retrograde direction.

The activity sphere of Saturn is

$$
m_{s}^{0.4} * a_{s}=0.0382 * 9.555 A U=0.365 A U=5.5 * 10^{7} \mathrm{~km}
$$

Most retrograde orbits are located about half of this value.

Suppose a prograde moon moves in a circular orbit with $a=0.2 A U$, the orbital velocity is

$$
V_{m}=\sqrt{m_{s} / 0.2} * V_{e}=1.13 \mathrm{~km} / \mathrm{sec}
$$

A particle with $a=a_{s}$ and $e<0.038$ moves on saturn's orbital plane would come into the activity sphere. Saturn's orbital velocity is $9.65 \mathrm{~km} / \mathrm{sec}$, an orbit with $e=0.02$ at perihelion $\left(q=a_{s}-0.2 A U\right)$ would have a relative velocity (to Saturn) about $0.2 \mathrm{~km} / \mathrm{sec}$. When this particle arrives into activity sphere at $r=0.2 A U$, its velocity is about $1.15 \mathrm{~km} / \mathrm{sec}$. A little more than the circular orbital velocity but on a retrograde direction.

If this "retrograde" body collided by the prograde satellite (in different orientations), their relative velocity can be reduced, and one of them could become a retrograde satellite.

\section{Collision probability}

Suppose we have two particles with $\mathrm{r}=10 \mathrm{~km}$. One particle moves in Saturn's activity sphere and another moves as a quasi-satellite around Saturn. When a quasi-satellite comes into Saturn's activity sphere, in one revolution, the collision probability is

$$
p_{\text {revo }}=(2 r)^{3} / R_{\text {act }}^{3}=5 * 10^{-19}
$$

where $R_{a c t}=0.365 A U=5.5 * 10^{7} \mathrm{~km}$
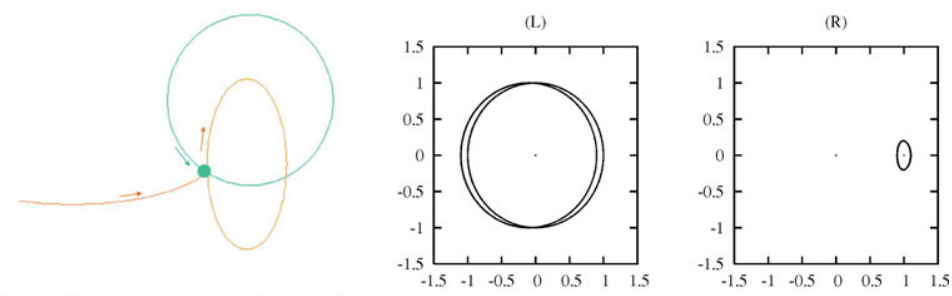

Figure 2. Sketch of a capture process

Figure 3. Quasi-satellite 
Table 2. First results from simulation

\begin{tabular}{ccccc}
\hline Test & $c$ & $a\left(10^{7} \mathrm{~km}\right)$ & $i\left(^{\circ}\right)$ & $e$ \\
\hline 1 & 0.2 & 1.895 & 171.3 & 0.159 \\
1 & 0.2 & 2.121 & 176.3 & 0.122 \\
1 & 0.2 & 2.154 & 170.6 & 0.085 \\
1 & 0.2 & 2.429 & 179.5 & 0.198 \\
2 & 0.5 & 2.620 & 169.0 & 0.419 \\
2 & 0.5 & 2.936 & 167.1 & 0.405
\end{tabular}

At the planetary and satellite system formation time, there are billions small bodies (Titan is about $1.3 \mathrm{E} 8$ greater than a $\mathrm{r}=10 \mathrm{~km}$ small body) .

The number of collisions should be multiplied by

$$
N_{\text {particle } 1} * N_{\text {particle } 2} * N_{\text {revolution }} \text {. }
$$

Let't put

$$
N_{\text {particle } 1}=1 * 10^{8}, N_{\text {particle } 2}=1 * 10^{9}, N_{\text {revolution }}=T / 30 y r=3 * 10^{7} / 30=1 * 10^{6},
$$
Then

$$
N_{\text {collision }}=p * N_{\text {particle } 1} * N_{\text {particle } 2} * N_{\text {revolution }}=5 * 10^{4}
$$

Of course this is not very accurate, but not very far from real process.

\section{Simulation}

In simulation, we set a planet as Saturn revolves around the Sun. Put $10^{n}(n=5)$ particles around Saturn with random orbital elements (small $i, e$ in Saturn's activity sphere, put $10 \cdot 10^{n}$ particles (quasi-satellites) move in similar orbits as Saturn but with $0<e<0.038$. We check the condition for impacts (collision coefficient $c=0.2-0.5$, $\Delta V_{\text {new }}=c \cdot \Delta V_{\text {old }}$ along the centers of two bodies) and obtain a few retrograde satellite orbits (They are all from quasi-satellites). We will simulate Large $N$ in order to obtain more results.

\section{Acknowledgements}

We would like to acknowledge the support of the National Natural Science Foundation of China (No. 10573037, 10933004), the exchange program between Finnish Academy and NSFC and the Minor Planetary Foundation of Purple Mountain Observatory.

\section{Reference}

Zheng, J. Q. 1994, ApJ Suppl., 108, 1994 